\title{
CLINICAL STUDY OF ACUTE PANCREATITIS AND ITS COMPLICATIONS
}

\author{
B. Raj Siddharth', Anil Kumar², Sindhu3, Satish ${ }^{4}$
}

${ }_{1}^{1}$ Associate Professor, Department of General Surgery, MGM Hospital, Warangal.

${ }^{2}$ Assistant Professor, Department of General Surgery, MGM Hospital, Warangal.

${ }^{3}$ Post Graduate, Department of General Surgery, MGM Hospital, Warangal.

${ }^{4}$ Post Graduate, Department of General Surgery, MGM Hospital, Warangal.

ABSTRACT

\section{BACKGROUND}

More than a century after its comprehensive description, acute pancreatitis remains a common disorder with devastating consequences. The presentation of wide spectrum of symptoms gives the clinician a heart breaking exercise to bring back the patient from the clutches of the disease process. Pancreatitis by itself is a disease which is unique, protean and extrudes into the diagnostic arena. It cannot be too strongly emphasized that the primary treatment of acute pancreatitis is conservative only, but it is the Pandora's box of manifestation with its inherent complications from surgery comes into play as diagnostic, prognostic and therapeutic endeavour. To study age and sex incidence of acute pancreatitis, to study and analyse the etiopathological factors associated with acute pancreatitis, especially alcohol aetiology in our study. To analyse the prognosis of acute pancreatitis using CT scan abdomen. To analyse the complications, to analyse the role of surgery in acute pancreatitis.

\section{METHODS}

This study was carried out on patients admitted in the General Surgery Department, MGM Hospital, from December 2013 to June 2015.

\section{RESULTS}

The incidence of acute pancreatitis was found to be in a younger age group in our study. Serum amylase and lipase both ( $80 \%$ sensitivity) should be used for diagnosis wherever possible. Ideally, all cases should be stratified during the first 48 hours according to one of the scoring systems (Balthazar's). Scoring systems help to identify patients who are more likely to have a severe attack and they should be referred to higher centres if adequate facilities are not available.

\section{KEYWORDS}

Serum Amylase, Pancreatitis, Serum Lipase.

HOW TO CITE THIS ARTICLE: Siddharth BR, Kumar A, Sindhu, et al. Clinical study of acute pancreatitis and its complications. J. Evolution Med. Dent. Sci. 2016;5(20):1053-1062, DOI: 10.14260/jemds/2016/245

\section{INTRODUCTION}

More than a century after its comprehensive description, acute pancreatitis remains a common disorder with devastating consequences. ${ }^{1}$ The presentation of wide spectrum of symptoms gives the clinician a heart breaking exercise to bring back the patient from the clutches of the disease process. Pancreatitis by itself is a disease, which is unique, protean and extrudes into the diagnostic arena. It cannot be too strongly emphasized that the primary treatment of acute pancreatitis is conservative only, but it is the Pandora's box of manifestation with its inherent complications from surgery comes into play as diagnostic, prognostic and therapeutic endeavour.

Because of the frequent emergency, multi-modality presentation, difficult pre-operative diagnosis and management of complications, this challenging subject is taken up for the present study in which we will be studying the clinical profile, management and complications of acute pancreatitis in our hospital. In spite of technical advances in medical and surgical fields, acute pancreatitis remains a major cause of morbidity and mortality.2,3

Financial or Other, Competing Interest: None.

Submission 06-02-2016, Peer Review 15-02-2016,

Acceptance 22-02-2016, Published 10-03-2016.

Corresponding Author:

Dr. Raj Siddharth,

MGM Hospital, Warangal.

E-mail: dr.rajsiddharth@gmail.com

DOI: $10.14260 /$ jemds/2016/245
Acute Pancreatitis (AP), defined as the acute nonbacterial inflammatory condition of the pancreas is derived from the early activation of digestive enzymes found inside the acinar cells with variable compromise of the gland itself, nearby tissues and other organs. AP is a disease with extremely different clinical expressions. Most patients suffer a mild and limited disease, but about one-fifth of cases develop Multiple Organ Dysfunction Syndrome (MODS), accompanied by high mortality. This great variability in presentation, clinical course and complications has given rise to the confusion related to AP related terminology. However, consensus meetings (Atlanta and later working groups) have provided more uniform definitions. ${ }^{4}$

For the last 25 years, there has been a global increase in incidence of AP along with many advances in diagnosis and treatment. However, progress in knowledge of its pathogenesis is scarce.

\section{AIMS AND OBJECTIVES OF THE STUDY}

- To study age and sex incidence of acute pancreatitis.

- To study and analyse the etiopathological factors associated with acute pancreatitis, especially alcohol aetiology in our study.

- To analyse the prognosis of acute pancreatitis using CT scan abdomen. ${ }^{5}$

- To analyse the complications.

- To analyse the role of surgery in acute pancreatitis. 


\section{MATERIALS AND METHODS}

Data was collected and processed using Excel software programmes. Observations are represented as bar diagrams and pie charts.

\section{CASE SHEET 1}

\begin{tabular}{|l|l|}
\hline Name : XXXX & I.P. No. : 7015 \\
\hline Age $: 44$ & D.O.A. : 12-01-2014 \\
\hline Sex : M & D.O.O. \\
\hline Address : Rangampet & D.O.D. : 22-01-2014 \\
\hline
\end{tabular}

Chief Complaints: Pain Abdomen since One Day.

History of Presenting Complaints

Patient was apparently asymptomatic one day before, then he developed pain abdomen initially in epigastric region radiating to back, then diffuse in nature associated with vomiting, nausea and anorexia. History of fever present since one day. History of distension of abdomen since one day.

\section{Past History}

No h/o similar complaints in the past.

No h/o Diabetes Mellitus, Hypertension, Tuberculosis, Previous Surgery, Drug Allergy, Gall Stones, Trauma.

\section{Family History}

No h/o similar complaints in the family.

\section{Personal History}

Diet-Mixed, Appetite-Normal, Bowel/Bladder-regular,

Sleep-Adequate, Habits-chronic alcoholic-since 20 years, daily $100 \mathrm{~mL}$, cheap liquor.

\section{General Physical Examination}

No Pallor, Icterus, Clubbing, Cyanosis, Koilonychia, Generalised Lymphadenopathy, Oedema.

\section{Vital Data}

Pulse Rate-98/min, Blood Pressure-100/60 mmHg.

Respiratory Rate-30/min, Temperature-Febrile.

\section{Systemic Examination}

\section{Per Abdomen}

Inspection

Shape of Abdomen-distended, Umbilicus-normal size and shape. All quadrants of abdomen moving with respiration. No swelling. Skin over abdomen-normal. No engorged veins. No visible pulsations. Hernial orifices-normal. Renal anglenormal. Spine-normal.

Palpation: No local rise of temperature, tenderness present all over the abdomen, guarding present, rigidity present. No organomegaly.

Percussion: Dull.

Auscultation: No bowel sounds.

Per Rectal Examination: Normal.

Examination of Other Systems:

Heart $-s_{1} s_{2}$ normal. Lungs-clear.

Provisional Diagnosis: Acute pancreatitis.

Differential Diagnosis: Hollow viscus perforation.

\section{Investigations}

Blood $\mathrm{Hb} \%$ - 11.0gms\%, TC- 12,400 cells/mm, neutrophils$76 \%$.

RBS- 98 mg/dL, Blood Urea-52 mg/dL, Serum Creatinine$1.1 \mathrm{mg} / \mathrm{dL}$.

Serum Amylase- 397 IU/L. Serum Lipase-1560 IU/L.

Urine Examination: Normal.

\section{Radiology and Sonography}

$\mathrm{X}$-ray Erect Abdomen-No pneumoperitoneum. Chest X-ray normal.

Ultrasonography of the abdomen and pelvis- Pancreas bulky in size with hypoechoic in echotexture.

CT scan of Abdomen- Pancreas appears bulky in size with decreased attenuation.

Peripancreatic collection noted s/o Acute Pancreatitis.

Balthazar Score: 2

\section{Final Diagnosis: Acute Pancreatitis}

\section{Treatment}

Conservative Management

Follow up: No complications.

\section{CASE SHEET 2}

\begin{tabular}{|l|l|}
\hline Name : XXXX & I.P. No. : 11256 \\
\hline Age : 56 & D.O.A. : 23-02-2014 \\
\hline Sex : F & D.O.O. \\
\hline Address: Rangampet & D.O.D. : 05-03-2014 \\
\hline
\end{tabular}

Chief Complaints: Pain abdomen since 3 days.

\section{History of Presenting Complaints}

Patient was apparently asymptomatic 3 days before, then she developed pain abdomen initially in epigastric region radiating to back, then diffuse in nature associated with vomiting, nausea and anorexia. No history of fever since 2 days. History of distension of abdomen since 1 day. No h/o constipation.

Past History: No h/o similar complaints in the past. H/o Diabetes Mellitus since 4 years, on medication. $\mathrm{H} / \mathrm{o}$ hysterectomy 20 years back.

No h/o Hypertension, Tuberculosis, Drug allergy, Gall Stones, Trauma.

Family History: No h/o similar complaints in the family.

Personal History

Diet-mixed, Appetite-normal, Bowel/Bladder-regular.

Sleep-adequate, addictions-nil.

\section{General Physical Examination}

No Pallor, Icterus, Clubbing, Cyanosis, Koilonychia, Generalised Lymphadenopathy, Oedema.

\section{Vital Data}

Pulse Rate-82/min, Blood Pressure-130/80 mmHg, Respiratory Rate-24/min, Temperature-Afebrile.

\section{Systemic Examination}

\section{Per Abdomen}

Inspection:

Shape of Abdomen-distended, Umbilicus-normal size and shape. All quadrants of abdomen moving with respiration. No swelling. Skin over abdomen-normal. No engorged veins, scar present. No visible pulsations. Hernial orifices-normal, Renal angle-normal, Spine-normal.

\section{Palpation:}

No local rise of temperature, tenderness present all over the abdomen, guarding present, rigidity present. No organomegaly.

Percussion: Dull.

Auscultation: No bowel sounds.

Per Rectal Examination: Normal.

Examination of Other Systems:

Heart $-\mathrm{s}_{1} \mathrm{~s}_{2}$ normal. Lungs-clear.

Provisional Diagnosis: Acute pancreatitis. 
Differential Diagnosis: Hollow viscus perforation. Investigations

Blood $\mathrm{Hb} \%$ - 9.0 gms\%, TC- 14,800 cells/mm, neutrophils$80 \%$.

RBS- 160 mg/dL, Blood Urea- 45 mg/dL, Serum Creatinine$1.4 \mathrm{mg} / \mathrm{dL}$.

Serum Amylase- 397 U/L,Serum lipase- 1560 U/L

Urine Examination: Normal.

Radiology and Sonography

X-ray Erect Abdomen- no pneumoperitoneum. Chest X-RayBronchitis.

Ultrasonography of the abdomen and pelvis- pancreas bulky in size with hypoechoic in echotexture with minimal collection.

CT scan of abdomen- pancreas appears bulky in size with peripancreatic fat stranding. Well defined collection noted in the head of the pancreas s/o Acute Pancreatitis.

Balthazar Score: 2

Final Diagnosis: Acute Pancreatitis

Treatment

Conservative Management

Follow up: No complications.

Case Sheet 3

\begin{tabular}{|l|l|}
\hline Name : XXXX & I.P. No. : 9302 \\
\hline Age : 39 & D.O.A. : 23-01-2014 \\
\hline Sex : M & D.O.O. \\
\hline Address : Parvathagiri & D.O.D. : 05-02-2014 \\
\hline
\end{tabular}

Chief Complaints: Pain Abdomen since One Week.

\section{History of Presenting Complaints}

Patient was apparently asymptomatic 1 week before, then he developed pain abdomen initially in epigastric region radiating to back, then diffuse in nature associated with vomiting, nausea and anorexia. History of fever since 3 days. History of distension of abdomen and constipation since 2 days.

Past History: No h/o similar complaints in the past.

H/o Hypertension since 2 years, on medication.

No h/o Diabetes mellitus, Tuberculosis, Drug allergy, Gall stones, Trauma, Previous surgery.

Family History: No h/o similar complaints in the family.

\section{Personal History}

Diet-mixed, Appetite-normal, Bowel/Bladder-regular,

Sleep-adequate, addictions-chronic alcoholic since 15 years, $200 \mathrm{~mL}$ daily, Gudumba.

General Physical Examination: Icterus present.

No Pallor, Clubbing, Cyanosis, Koilonychia, Generalised Lymphadenopathy, Oedema.

\section{Vital Data}

Pulse Rate-112/min, Blood Pressure-90/60 mmHg,

Respiratory Rate-32/min, Temperature-febrile.

Systemic Examination

\section{Per Abdomen}

\section{Inspection:}

Shape of Abdomen-distended, Umbilicus-normal size and shape. No swelling. Skin over abdomen-normal. No engorged veins. No scar. No visible pulsations. Hernial orifices-normal. Renal angle-normal. Spine-normal.

\section{Palpation:}

No local rise of temperature, tenderness present all over the abdomen, guarding present, rigidity present. No organomegaly.
Percussion: Dull note in the flanks.

Auscultation: No bowel sounds.

Per Rectal Examination: Normal.

Examination of Other Systems:

Heart $-\mathrm{s}_{1} \mathrm{~S}_{2}$ normal, lungs-B/L airway entry decreased.

Provisional Diagnosis: Acute pancreatitis.

Differential Diagnosis: Hollow viscus perforation.

Investigations

Blood $\mathrm{Hb} \%-12.0$ gms\%, TC-28,110 cells/mm, neutrophils92\%.

RBS-110 mg/dL, Blood Urea-68 mg/dL.

Serum Creatinine-1.9 mg/dL, Serum Amylase-1983 IU/L, Serum lipase-672 IU/L.

Urine Examination: Normal.

Radiology and Sonography

X-ray Erect Abdomen-no pneumoperitoneum. Chest X-RayBronchitis.

Ultrasonography of the abdomen and pelvis-pancreas oedematous and bulky in size with hypoechoic in echotexture with free fluid in pelvis.

CT scan of Abdomen-pancreas appears bulky in size with decreased attenuation, on contrast multiple unenhancing areas noted s/o necrosis with free fluid in the pelvis.

Acute necrotizing pancreatitis.

Balthazar Score: 8

Final Diagnosis: Acute Necrotizing Pancreatitis

Treatment

Conservative Management: To resuscitate the patient $\mathrm{f} / \mathrm{b}$ necrosectomy under general anaesthesia. Patient died in spite of resuscitation.

\section{CASE SHEET 4}

\begin{tabular}{|l|l|}
\hline Name : XXXX & I.P. No. : 2456 \\
\hline Age : 22 & D.O. A. : 03-01-2014 \\
\hline Sex : F & D.0.0.: 05-01-2014 \\
\hline Address : Jangoan & D.O.D. : 10-01-2014 \\
\hline
\end{tabular}

Chief Complaints: Pain abdomen since one day.

History of Presenting Complaints

Patient was apparently asymptomatic 1 day before, then she developed pain abdomen initially in epigastric and right hypochondriac region radiating to back, then diffuse in nature associated with vomiting, nausea and anorexia, dyspepsia. History of fever since 1 day. History of distension of abdomen and constipation since 1 day.

Past History: No h/o similar complaints in the past.

H/o cholelithiasis. No h/o Diabetes Mellitus, Hypertension, Tuberculosis, Drug allergy, Trauma, Previous Surgery.

Family History: No h/o similar complaints in the family.

\section{Personal History}

Diet-Mixed, Appetite-Normal, Bowel/Bladder-Regular,

Sleep-Adequate, Addictions-nil.

General Physical Examination: Icterus present.

No Pallor, Clubbing, Cyanosis, Koilonychia, Generalised Lymphadenopathy, Oedema.

\section{Vital Data}

Pulse Rate - 82/min, Blood Pressure-110/70 mmHg, Respiratory Rate $-22 / \mathrm{min}$, Temperature-febrile.

Systemic Examination

Per Abdomen

Inspection:

Shape of Abdomen-distended, Umbilicus-normal size and shape. No swelling. Skin over abdomen-normal. 
No engorged veins. No scar. No visible pulsations. Hernial orifices-normal.

Renal angle-normal, Spine-normal.

\section{Palpation:}

No local rise of temperature, tenderness present all over the abdomen, guarding present, rigidity present, no organomegaly.

Percussion: Dull note noted.

Auscultation: Sluggish bowel sounds.

Per Rectal Examination: Normal.

Examination of Other Systems:

Heart -S1 S2 normal. Lungs-B/L airway entry decreased.

Provisional Diagnosis: Acute pancreatitis.

Differential Diagnosis: Hollow viscus perforation.

Investigations:

Blood $\mathrm{Hb} \%$ - 10.0 gms\%, TC- 15,100 cells/mm, neutrophils$72 \%$.

RBS- $100 \mathrm{mg} / \mathrm{dL}$, Blood Urea-38 mg/dL, Serum Creatinine$1.2 \mathrm{mg} / \mathrm{dL}$, Serum Amylase- $984 \mathrm{IU} / \mathrm{L}$, Serum lipase-185 IU/L. LFT-normal.

Urine Examination: Normal.

Radiology and Sonography

X-ray Erect Abdomen-no pneumoperitoneum. Chest X-ray normal.

Ultrasonography of the abdomen and pelvis-pancreas bulky in size with hypoechoic in echotexture.

Gall bladder - multiple stones largest measuring $7 \mathrm{~mm}$ noted.

CT scan of Abdomen-pancreas appears bulky in size with decreased attenuation.

Peripancreatic collection noted s/o Acute Pancreatitis.

Pancreatic duct dilated.

Balthazar Score: 1

Final Diagnosis: Acute Pancreatitis due to Gall Stones.

Treatment

Conservative Management: F/b laparoscopic cholecystectomy.

\section{CASE SHEET 5}

\begin{tabular}{|l|l|}
\hline Name : XXXX & I.P. No. : 3471 \\
\hline Age $: 24$ & D.O. A. : 11-05-2014 \\
\hline Sex : M & D.O.0. : 26-06-2014 \\
\hline Address : Parkal & D.O.D. : 03-07-2014 \\
\hline
\end{tabular}

Chief Complaints: Pain abdomen since one day.

\section{History of Presenting Complaints}

Patient was apparently asymptomatic 1 week before, then he developed pain abdomen initially in epigastric region radiating to back, then diffuse in nature associated with vomiting, nausea and anorexia. History of fever and distension of abdomen since 1 day.

Past History: No h/o similar complaints in the past.

No h/o Diabetes Mellitus, Hypertension, Tuberculosis, Drug Allergy, Gall Stones, Trauma, Previous Surgery.

Family History: No h/o similar complaints in the family.

\section{Personal History}

Diet-mixed, Appetite-normal, Bowel/Bladder-regular, Sleep -adequate, addictions-chronic alcoholic, $150 \mathrm{~mL}$ daily, cheap liquor.

General Physical Examination

No Pallor, Icterus, Clubbing, Cyanosis, Koilonychia, Generalised Lymphadenopathy, Oedema.

\section{Vital Data}

Pulse Rate - 102/min, Blood Pressure-100/70 mmHg, Respiratory Rate $-25 / \mathrm{min}$, Temperature-febrile.

Systemic Examination

Per Abdomen

Inspection:

Shape of Abdomen-distended, Umbilicus-normal size and shape. No swelling. Skin over abdomen-normal. No engorged veins. No scar. No visible pulsations. Hernial orificesnormal. Renal angle-normal. Spine-normal.

Palpation: No local rise of temperature, tenderness present all over the abdomen, guarding present, rigidity present. No organomegaly.

Percussion: No e/o free fluid.

Auscultation: No bowel sounds.

Per Rectal Examination: Normal.

Examination of Other Systems:

Heart $-\mathrm{s}_{1} \mathrm{~s}_{2}$ normal. Lungs-B/L airway entry present.

Provisional Diagnosis: Acute pancreatitis.

Differential Diagnosis: Hollow viscus perforation.

Investigations

Blood Hb\%-12.0 gms\%, TC- 14500 cells/mm, neutrophils$72 \%$.

RBS- $120 \mathrm{mg} / \mathrm{dL}$, Blood Urea-32 mg/dL, Serum Creatinine$1.0 \mathrm{mg} / \mathrm{dL}$,Serum Amylase- $563 \mathrm{IU} / \mathrm{L}$.

Serum lipase-1154 IU/L.

Urine Examination: Normal.

Radiology and Sonography

X-ray Erect Abdomen-no pneumoperitoneum. Chest X-raynormal.

Ultrasonography of the abdomen and pelvis-pancreas bulky in size with hypoechoic in echotexture with collection in the tail of the pancreas.

CT scan of Abdomen-pancreas are bulky in size with decreased attenuation with multiple collections noted in the tail of pancreas.

S/o Acute Pancreatitis.

Balthazar Score: 5

Final Diagnosis: Acute Pancreatitis.

Treatment

Conservative Management: Follow up: Patient developed pseudocyst of pancreas and was operated (cystogastrostomy).

\section{CASE SHEET 6}

\begin{tabular}{|l|l|}
\hline Name : XXXX & I.P. No. : 3783 \\
\hline Age $: 42$ & D.O. A. : 04-03-2014 \\
\hline Sex : M & D.O.O. : \\
\hline Address: Rangasaipet & D.O.D. : 12-03-2014 \\
\hline
\end{tabular}

Chief Complaints: Pain abdomen since one day.

History of Presenting Complaints

Patient was apparently asymptomatic one day, before then he developed pain abdomen initially in epigastric region radiating to back, then diffuse in nature associated with vomiting, nausea and anorexia. History of fever present and distension of abdomen since 1 day.

Past History: No h/o similar complaints in the past.

No h/o Diabetes Mellitus, Hypertension, Tuberculosis, Previous Surgery, Drug Allergy, Gall Stones, Trauma.

Family History: No h/o similar complaints in the family. 


\section{Personal History}

Diet-mixed, Appetite-normal, Bowel/Bladder-regular, Sleep-adequate, Habits-chronic alcoholic $100 \mathrm{~mL}$ daily since 10 years, cheap liquor.

General Physical Examination

No Pallor, Icterus, Clubbing, Cyanosis, Koilonychia, Generalised Lymphadenopathy, Oedema.

\section{Vital Data}

Pulse Rate-96/min, Blood Pressure-90/60 mmHg, Respiratory Rate-22/min, Temperature-Febrile.

Systemic Examination

Per Abdomen

Inspection:

Shape of Abdomen-distended, Umbilicus-normal size and shape. All quadrants of abdomen moving with respiration. No swelling. Skin over abdomen-normal. No engorged veins. No visible pulsations. Hernial orifices-normal. Renal anglenormal. Spine-normal.

Palpation: No local rise of temperature, tenderness present all over the abdomen, guarding present, rigidity present. No organomegaly.

Percussion: Resonant.

Auscultation: No bowel sounds.

Per Rectal Examination: Normal.

Examination of Other Systems:

Heart $-\mathbf{s}_{1} \mathbf{s}_{2}$ normal. Lungs-clear.

Provisional Diagnosis: Acute pancreatitis.

Differential Diagnosis: Hollow viscus perforation.

\section{Investigations}

Blood $\mathrm{Hb} \%-12.0$ gms\%, TC-11,400 cells/mm, neutrophils$68 \%$.

RBS-108 mg/dL, Blood Urea-42 mg/dL, Serum

Creatinine- $1.0 \mathrm{mg} / \mathrm{dL}$.

Serum Amylase-464 IU/L, Serum lipase-700 IU/L.

Urine Examination: Normal.

Radiology and Sonography

X-ray Erect Abdomen-no pneumoperitoneum. Chest X-raynormal.

Ultrasonography of the abdomen and pelvis-pancreas bulky in size with hypoechoic in echotexture.

CT scan of Abdomen-pancreas appears bulky in size with decreased attenuation.

Peripancreatic collection noted s/o Acute Pancreatitis.

Balthazar Score: 3

Final Diagnosis: Acute Pancreatitis.

Treatment

Conservative Management

Follow up: No complications.

\section{RESULTS AND OBSERVATIONS}

A total number of 105 patients were entered in the study. All had an admission diagnosis of acute pancreatitis.

\section{Age Distribution}

Median age of our study was 32 yrs.

\begin{tabular}{|c|c|c|}
\hline $\begin{array}{c}\text { Age } \\
\text { Groups }\end{array}$ & Frequency & Percentage \\
\hline$<=20$ & 8 & $7.6 \%$ \\
\hline $21-30$ & 36 & $34.2 \%$ \\
\hline $31-40$ & 36 & $34.2 \%$ \\
\hline $41-50$ & 15 & $14.2 \%$ \\
\hline $51-60$ & 10 & $9.5 \%$ \\
\hline \multicolumn{2}{|c|}{ Table 1: Age Distribution } \\
\hline
\end{tabular}

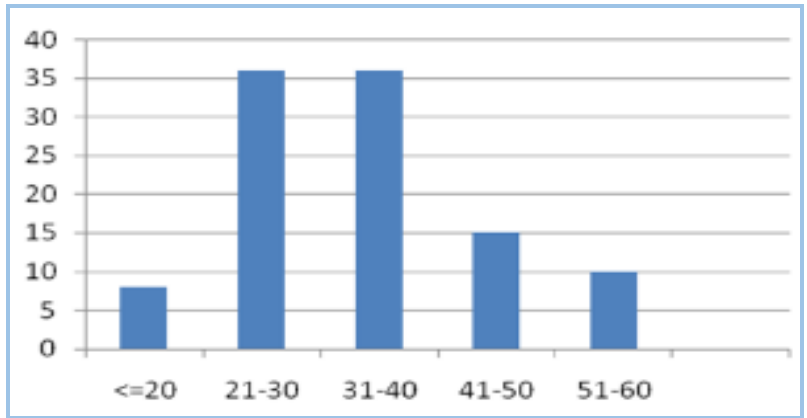

Fig. 1: Age Distribution

\section{Age Groups}

Out of 105 patients, 75 were males $(71.42 \%), 30$ females (28.58\%).

\begin{tabular}{|c|c|c|}
\hline Sex & Frequency & Percentage \\
\hline Male & 75 & $71.42 \%$ \\
\hline Female & 30 & $28.58 \%$ \\
\hline \multicolumn{3}{|c|}{ Table 2: Sex Distribution } \\
\hline
\end{tabular}

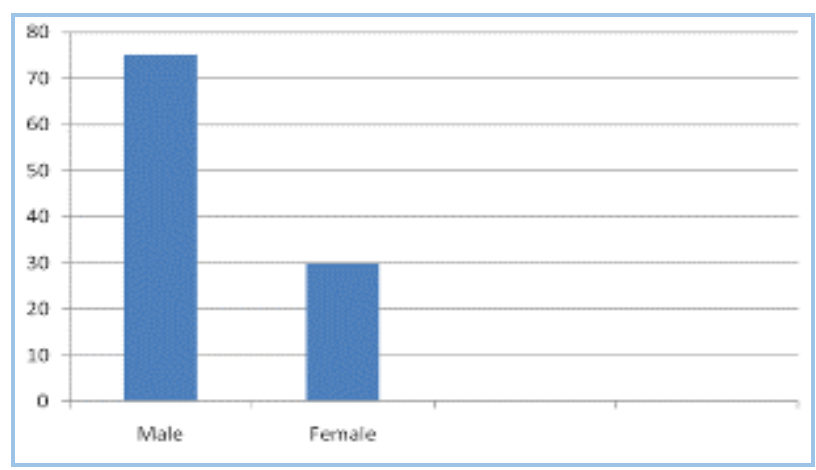

Fig. 2: Sex Distribution

\section{Aetiology}

Since our objective was mainly to study alcoholic aetiology; $69 \%$ of cases were of alcoholic aetiology. Of non-alcoholic aetiology, gall stones was most common.

In 4 cases, aetiology was not identified even after biochemical and radiological studies.

In a case, pancreatic biliary anomaly was the cause of pancreatitis in young male aged around 16 years.

In a case, Rifampicin was the cause of pancreatitis in a patient on ATT, which was confirmed by excluding drug from regimen.

\begin{tabular}{|c|c|c|}
\hline Aetiology & Frequency & Percentage \\
\hline Alcohol & 73 & $69.5 \%$ \\
\hline $\begin{array}{c}\text { Anomalous biliary pancreatic } \\
\text { junction }\end{array}$ & 1 & $0.9 \%$ \\
\hline Biliary stones & 26 & $24.7 \%$ \\
\hline Drug & 1 & $0.9 \%$ \\
\hline Idiopathic & 4 & $3.8 \%$ \\
\hline Table 3: Distribution of Aetiology \\
\hline
\end{tabular}

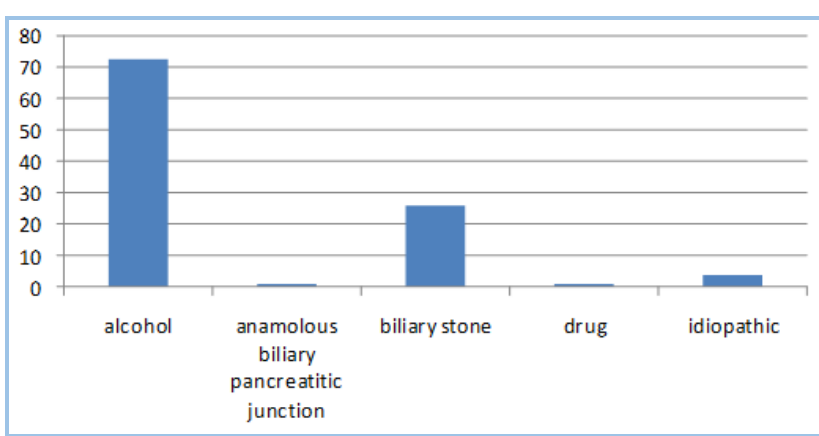

Fig. 3: Distribution of Aetiology 
Aetiology

\begin{tabular}{|c|c|c|}
\hline Symptom & Number & Percentage \\
\hline Pain abdomen & 105 & $100 \%$ \\
\hline Vomiting & 21 & $20 \%$ \\
\hline Distention & 40 & $38 \%$ \\
\hline Fever & 14 & $13.3 \%$ \\
\hline Anorexia & 46 & $43.8 \%$ \\
\hline Nausea & 54 & $51.4 \%$ \\
\hline \multicolumn{2}{|c|}{ Table 4: Clinical Presentation } \\
\hline
\end{tabular}

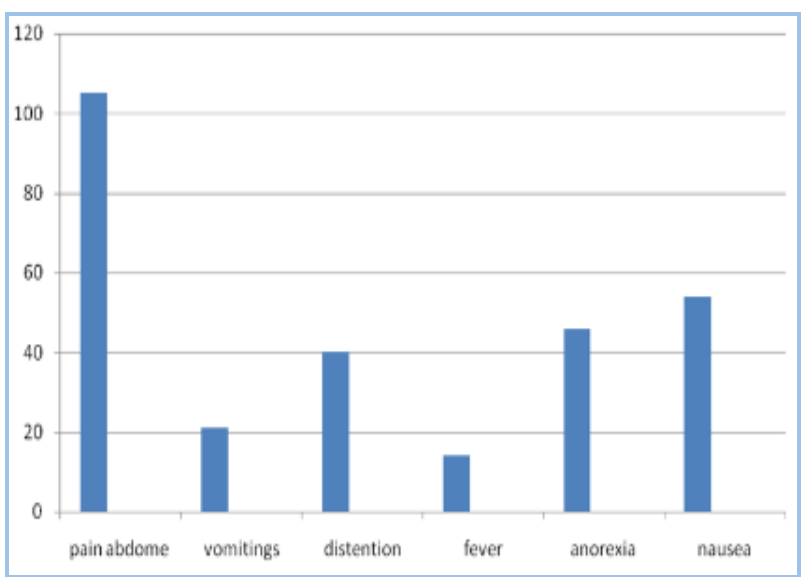

Fig. 4

Distribution of Serum Amylase Levels

\begin{tabular}{|c|c|c|}
\hline S. Amylase Levels (IU/L) & $\begin{array}{c}\text { No. of } \\
\text { Persons }\end{array}$ & Percentage \\
\hline $340-500$ & 25 & $23.80 \%$ \\
\hline $500-1000$ & 22 & $20.95 \%$ \\
\hline $1000-1500$ & 31 & $29.52 \%$ \\
\hline $1500-2000$ & 27 & $25.71 \%$ \\
\hline \multicolumn{3}{|c|}{ Table 5 } \\
\hline
\end{tabular}

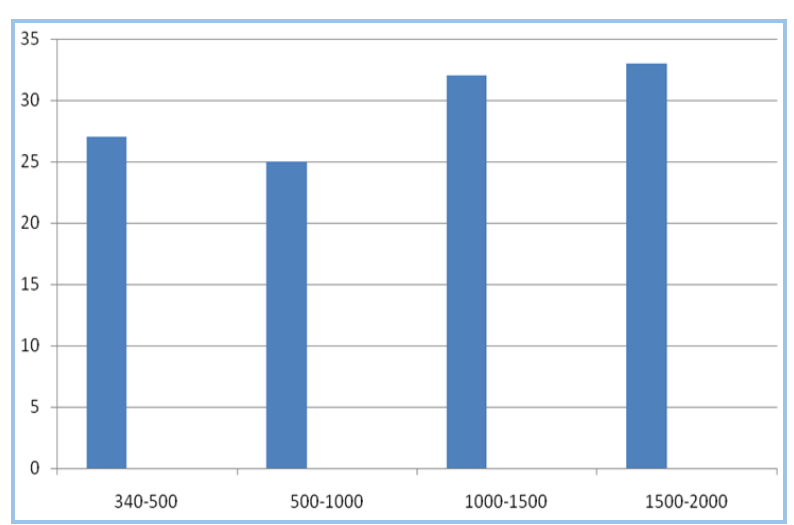

Fig. 5: Distribution of Serum Amylase Levels

\begin{tabular}{|c|c|c|}
\hline $\begin{array}{c}\text { Serum Lipase Levels } \\
\text { (IU/L) }\end{array}$ & $\begin{array}{c}\text { No. of } \\
\text { persons }\end{array}$ & Percentage \\
\hline $320-500$ & 23 & $21.90 \%$ \\
\hline $500-1000$ & 30 & $28.57 \%$ \\
\hline $1000-1500$ & 25 & $23.80 \%$ \\
\hline $1500-2000$ & 27 & $25.71 \%$ \\
\hline \multicolumn{2}{|c|}{ Table 6: Distribution of Serum Lipase Levels } \\
\hline
\end{tabular}

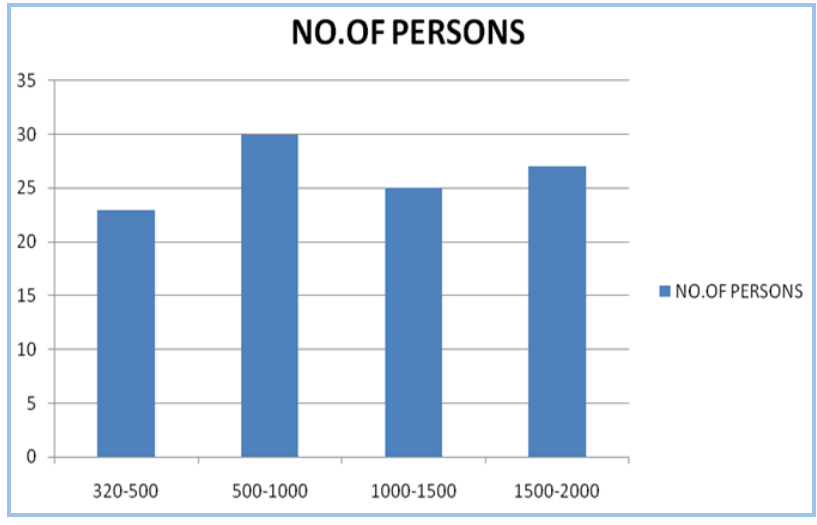

Fig. 6: Distribution of Serum Lipase Levels

Distribution Balthazar's Score (CT Comparison)

$56 \%$ of patients had Balthazar in between 0-3.

\begin{tabular}{|c|c|c|}
\hline Groups & Frequency & Percentage \\
\hline $0-3$ & 49 & $46.66 \%$ \\
\hline $4-6$ & 25 & $23.80 \%$ \\
\hline $7-10$ & 31 & $29.52 \%$ \\
\hline \multicolumn{2}{|c|}{ Table 7: Distribution of Balthazar's Score } \\
\hline
\end{tabular}

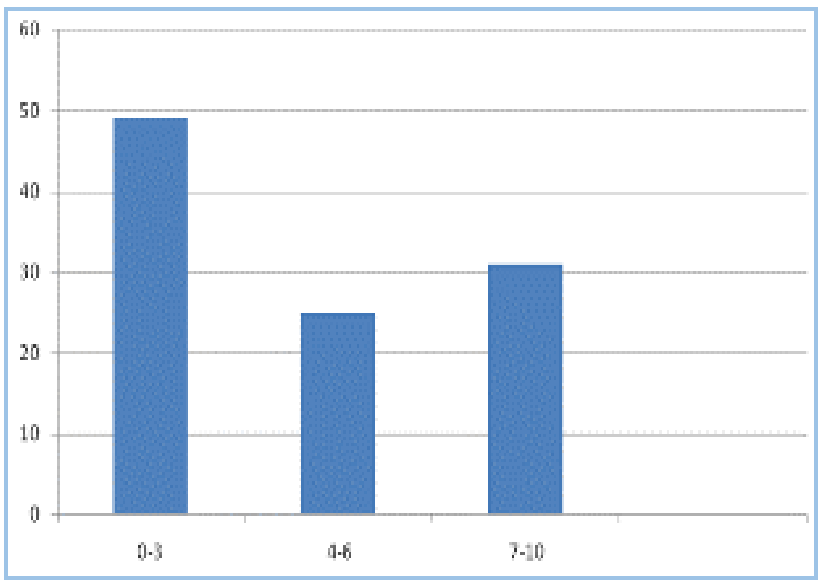

Fig. 7: Distribution of Balthazar's Score

\section{GROUPS}

\section{Local Complications}

Acute fluid collection was most common complication. One patient with necrosis had GDA aneurysm.

\begin{tabular}{|c|c|c|}
\hline Local Complications & Number & Percentage \\
\hline Acute fluid collection & 24 & $22.85 \%$ \\
\hline Pseudocyst & 16 & $15.23 \%$ \\
\hline Necrosis & 19 & $18.09 \%$ \\
\hline GDA aneurysm & 1 & $0.95 \%$ \\
\hline Faecal fistula & 2 & $1.90 \%$ \\
\hline Ascites & 1 & $0.95 \%$ \\
\hline \multicolumn{3}{|c|}{ Table 8: Distribution of Local Complications } \\
\hline
\end{tabular}




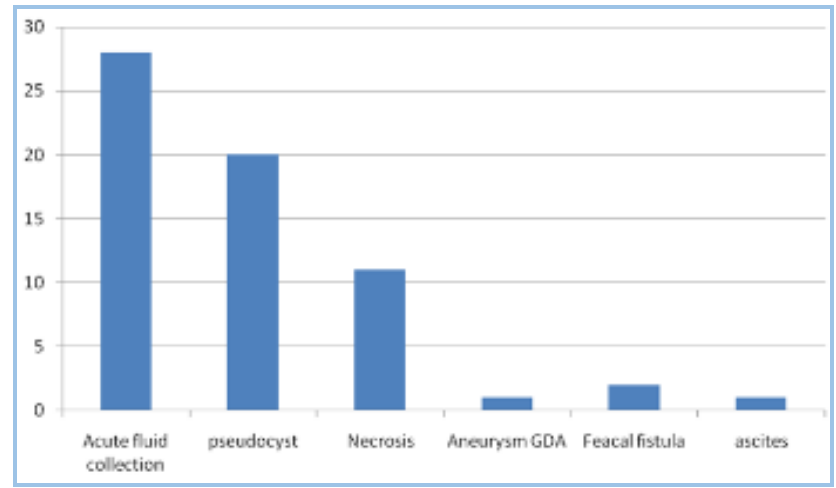

Fig. 8: Distribution of Local Complications

\section{LOCAL COMPLICATIONS}

\section{SYSTEMIC COMPLICATIONS}

Of systemic complications, left-sided pleural effusion was most common.

\begin{tabular}{|c|c|c|}
\hline Systemic Complications & Frequency & Percentage \\
\hline ARDS & 3 & $2.85 \%$ \\
\hline ARDS, Renal failure, Sepsis & 1 & $0.95 \%$ \\
\hline Left pleural effusion & 7 & $6.66 \%$ \\
\hline ARDS, Renal failure & 1 & $0.95 \%$ \\
\hline ARDS, Left pleural effusion & 1 & $0.95 \%$ \\
\hline \multicolumn{2}{|c|}{ Table 9: Distribution of Systemic Complications } \\
\hline
\end{tabular}

Venn diagram on systemic complications

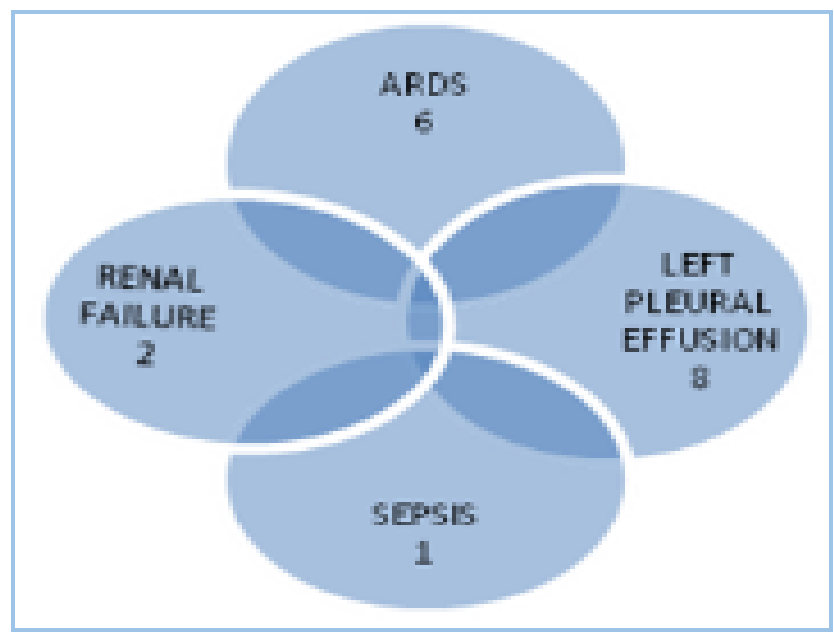

Fig. 9: Distribution of Systemic Complications $\mathrm{n}=13$

\section{Mortality}

Mortality in our study is 8 , out of which 3 is due to acute necrotizing pancreatitis.

One patient had 4 laparotomies for necrosectomy and faecal fistula.

\begin{tabular}{|c|c|c|c|}
\hline Reference & All Cases & Death Rate & $\begin{array}{c}\text { \% Death } \\
\text { Rate }\end{array}$ \\
\hline $\begin{array}{c}\text { Acute } \\
\text { pancreatitis }\end{array}$ & 105 & 08 & $7.6 \%$ \\
\hline ANP & 19 & 3 & $15.78 \%$ \\
\hline \multicolumn{3}{|c|}{$\begin{array}{r}\text { Table 10: Distribution of Mortality } \\
\text { from Acute Pancreatitis }\end{array}$} \\
\hline
\end{tabular}

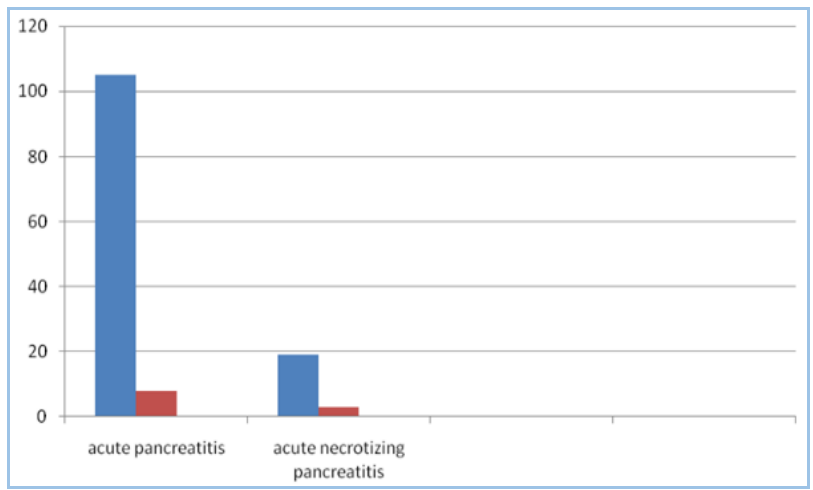

Fig. 10: Surgery in Acute Pancreatitis

Biliary pancreatitis cases without necrosis underwent Laparoscopic Cholecystectomy, either at time of discharge or 4-6 weeks after attack. Of 19 patients with necrosis, 10 underwent necrosectomy; after 3-4 weeks after attack patients with biliary pancreatitis underwent simultaneous cholecystectomy.

One patient underwent laparotomy for suspected peritonitis was found operatively to have necrotizing pancreatitis.

\begin{tabular}{|c|c|c|}
\hline Surgery & & Percentage \\
\hline Lap cholecystectomy & 8 & $7.61 \%$ \\
\hline Laparotomy and drainage & 1 & $0.95 \%$ \\
\hline Necrosectomy & 10 & $9.52 \%$ \\
\hline Cystogastrostomy & 5 & $4.76 \%$ \\
\hline \multicolumn{2}{|c|}{ Table 11: Surgery in Acute Pancreatitis } \\
\hline
\end{tabular}

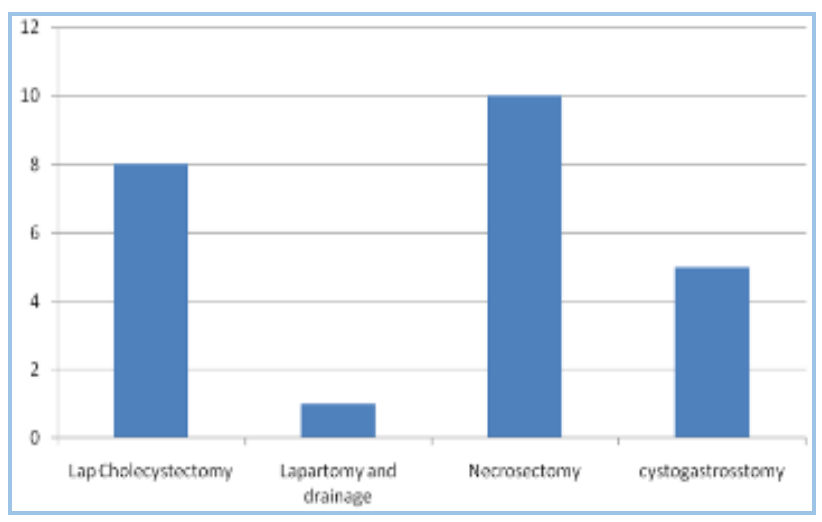

Fig. 11: Surgery in Acute Pancreatitis

\section{SURGERY TYPE}

Alcoholic Aetiology Vs. Other Aetiology

Alcohol as aetiology was common in males.

Death and necrosis was common in alcohol group compared to others.

\begin{tabular}{|c|c|c|c|c|c|}
\hline \multicolumn{6}{|c|}{ Comparison of Alcoholic Vs. Other Aetiology } \\
\hline & Frequency & Male & Female & Necrosis & $\begin{array}{c}\text { Death } \\
\text { rate }\end{array}$ \\
\hline Alcoholic & 73 & $100 \%$ & $0 \%$ & $20.54 \%$ & $8.21 \%$ \\
\hline $\begin{array}{c}\text { Other } \\
\text { aetiology }\end{array}$ & 32 & $6.25 \%$ & $93.75 \%$ & $12.5 \%$ & $6.25 \%$ \\
\hline \multicolumn{6}{|c|}{ Table 12: Alcoholic Aetiology Vs. Other Aetiology } \\
\hline
\end{tabular}




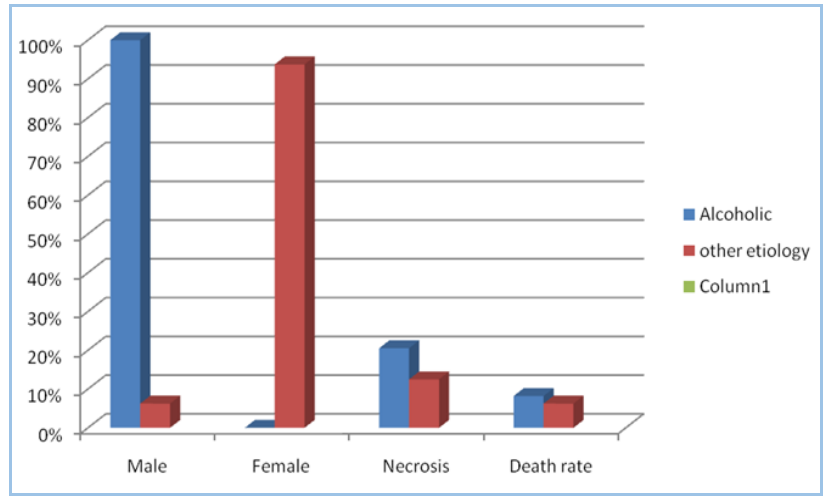

Fig. 12: Alcoholic Aetiology Vs. Other Aetiology

\section{DISCUSSION}

Patients with severe acute pancreatitis demand considerable resources in the form of imaging, endoscopy, surgery and intensive care. This study was conducted at MGM Hospital, Warangal.

\section{Sex Incidence}

In the present study, acute pancreatitis was found to be two and a half times more common in males than females. (MF 2.5:1). A prospective audit in 7 hospitals from South England (hereby referred to as South England Audit) also showed males more commonly affected (MF 1.32:1).

\begin{tabular}{|l|c|c|}
\hline & Our Study & SKC Toh et al. \\
\hline Sex Incidence & $2.5: 1$ & $1.32: 1$ \\
\hline Comparison of Sex Incidences-Our's Vs. Skc Toh et al. \\
\hline \multicolumn{3}{|c|}{ Table 13 } \\
\hline
\end{tabular}

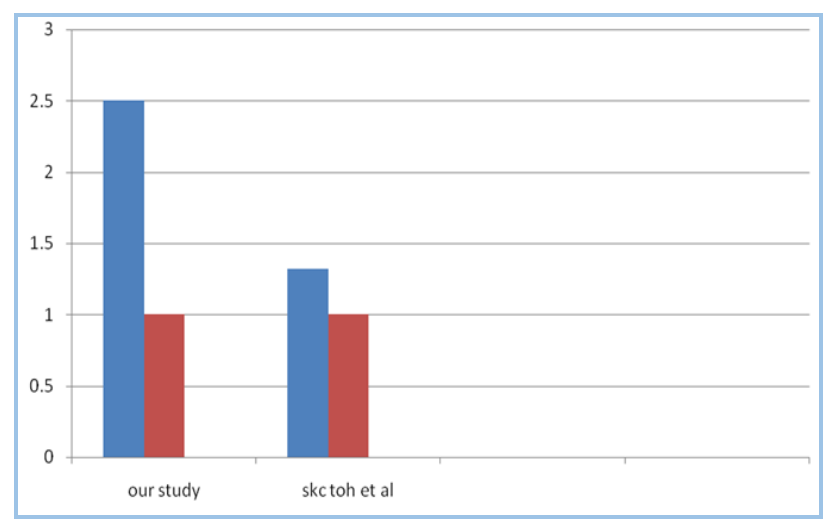

Fig. 13: Comparison of Sex Incidences -Our's Vs. SKC Toh et al.

Age Incidence

The peak incidence was in the $4^{\text {th }}$ decade of life - the most productive age group. The median age group in our study was 32 years compared to 54 years in the South England Audit, indicating a younger age group being affected.

\begin{tabular}{|c|c|c|}
\hline & Our Study & SKC Toh et al. Study. ${ }^{\mathbf{}}$ \\
\hline Median age & 32 & 54 \\
\hline \multicolumn{2}{|c|}{ Table 14: Comparison of Age Incidences } \\
-Our's Vs. SKC Toh et al. Studies \\
\hline
\end{tabular}

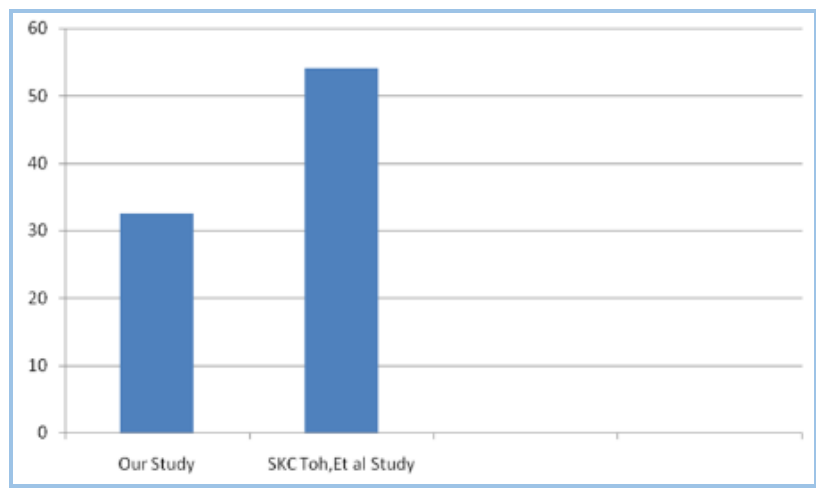

Fig. 14: Comparison of Age IncidencesOur's Vs. SKC Toh et al. Studies Aetiology

Since our objective was mainly to study alcoholic aetiology, at least $69 \%$ of cases were of alcoholic aetiology.

\begin{tabular}{|c|c|c|c|}
\hline Aetiology & $\begin{array}{c}\text { Our's } \\
\text { Study }\end{array}$ & $\begin{array}{c}\text { M Sekimoto, } \\
\text { et al. study. }\end{array}$ & $\begin{array}{c}\text { SKC Toh, et } \\
\text { al. studies.7 }\end{array}$ \\
\hline Alcohol & 69.52 & & $20 \%$ \\
\hline $\begin{array}{c}\text { Biliary } \\
\text { stones }\end{array}$ & $24.76 \%$ & $24 \%$ & $33 \%$ \\
\hline Drug & $0.95 \%$ & $1 \%$ & $8 \%$ \\
\hline Idiopathic & $3.8 \%$ & $23 \%$ & $32 \%$ \\
\hline Others & $0.95 \%$ & $52 \%$ & $15 \%$ \\
\hline Table 15: Comparison of Aetiology- Our's, M Sekimoto \\
et al. and SKC Toh et al. Studies \\
\hline
\end{tabular}

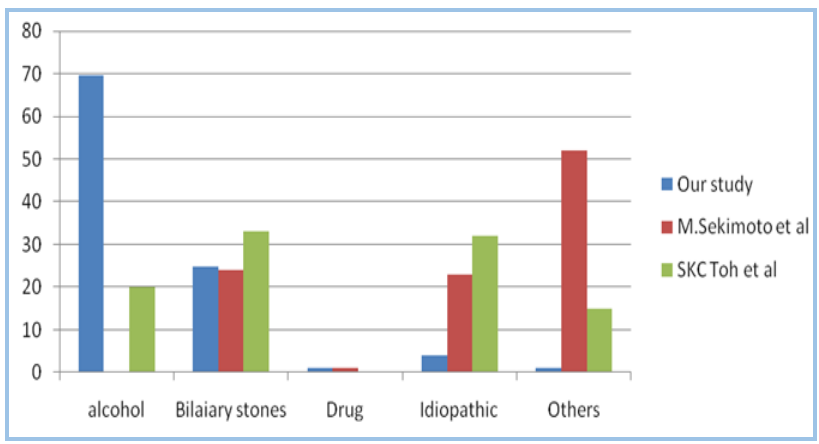

Fig. 15: Comparison of Aetiologies-

Our's, M Sekimoto et al. and SKC Toh et al. studies

Enteral Nutrition and Mortality in Acute Necrotizing Pancreatitis

Role of enteral nutrition was assessed in acute necrotizing pancreatitis only. Since study size was small and patients were not affordable for parenteral nutrition, no internal comparison was done.

Role of enteral nutrition was assessed in relation with mortality and operative intervention of our study and Doley RP, et al. study.

\begin{tabular}{|c|c|c|c|}
\hline & $\begin{array}{c}\text { \# of } \\
\text { Patients }\end{array}$ & $\begin{array}{c}\text { \% Death } \\
\text { Rate }\end{array}$ & $\begin{array}{c}\text { \% Operative } \\
\text { Intervention }\end{array}$ \\
\hline Our study & 19 & $15.7 \%$ & $52.6 \%$ \\
\hline $\begin{array}{c}\text { Doley RP, } \\
\text { et al. study }\end{array}$ & 25 & $20 \%$ & $56 \%$ \\
\hline \multicolumn{3}{|c|}{ Table 16: Enteral Nutrition, Mortality and } \\
Operative Intervention in ANP \\
\hline
\end{tabular}




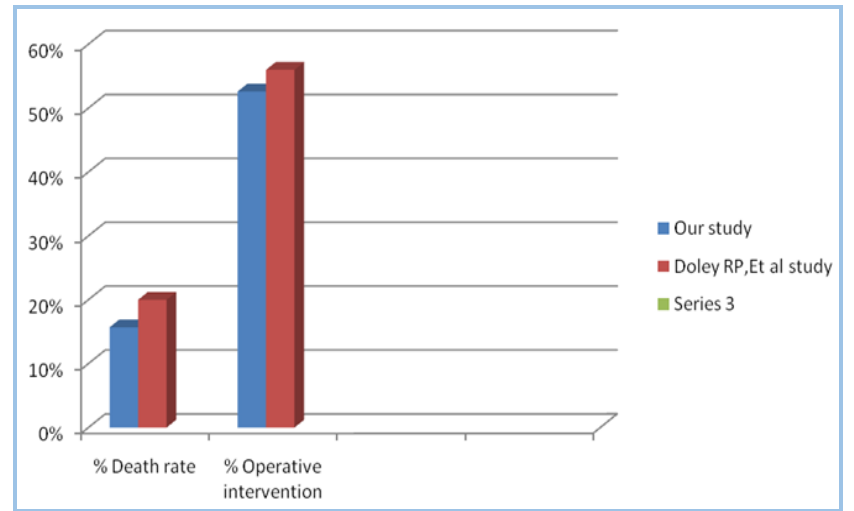

Fig. 16: Enteral Nutrition, Mortality and Operative Intervention in ANP

Role of prophylactic antibiotics was assessed in acute necrotizing pancreatitis only. Since study size was small, no internal comparison was done.

Antibiotics used in our study were inj. Ciprofloxacin and inj. Metronidazole, since it was available in hospital free of cost. Antibiotics was started immediately after necrosis was documented in CECT abdomen.

Role of prophylactic antibiotics was assessed in relation with mortality of our study and Pederzoli. ${ }^{8}$ Sainio. ${ }^{9}$ Delcenserie. ${ }^{10} \quad$ Schwarz. $^{11} \quad$ Nordback. ${ }^{12} \quad$ Isenmam. ${ }^{13}$ studies. ${ }^{14,15}$

\begin{tabular}{|c|c|c|c|}
\hline Reference & Agent & $\begin{array}{c}\text { Death } \\
\text { Rate }\end{array}$ & $\begin{array}{c}\text { \% } \\
\text { Death } \\
\text { Rate }\end{array}$ \\
\hline Pederzoli. ${ }^{8}$ & Imipenem & $3 / 41$ & $7 \%$ \\
\hline Sanion. & Cefuroxime & $1 / 30$ & $3 \%$ \\
\hline Delcenserie. 10 & $\begin{array}{c}\text { Ceftazidime, } \\
\text { amikacin, } \\
\text { metronidazole }\end{array}$ & $1 / 11$ & $9 \%$ \\
\hline Schwarz. $^{11}$ & $\begin{array}{c}\text { Ofloxacin, } \\
\text { metronidazole }\end{array}$ & $0 / 13$ & $0 \%$ \\
\hline Nordback. $^{12}$ & Imipenem, cilastatin & $2 / 25$ & $8 \%$ \\
\hline Isenmam. ${ }^{13}$ & $\begin{array}{c}\text { Ciprofloxacin, } \\
\text { metronidazole }\end{array}$ & $3 / 58$ & $5 \%$ \\
\hline Our Study & $\begin{array}{c}\text { Ciprofloxacin, } \\
\text { metronidazole }\end{array}$ & $\mathbf{3 / 1 9}$ & $\mathbf{1 5 . 7 \%}$ \\
\hline Table 17: Prophylactic Antibiotics and Mortality in ANP \\
\hline
\end{tabular}

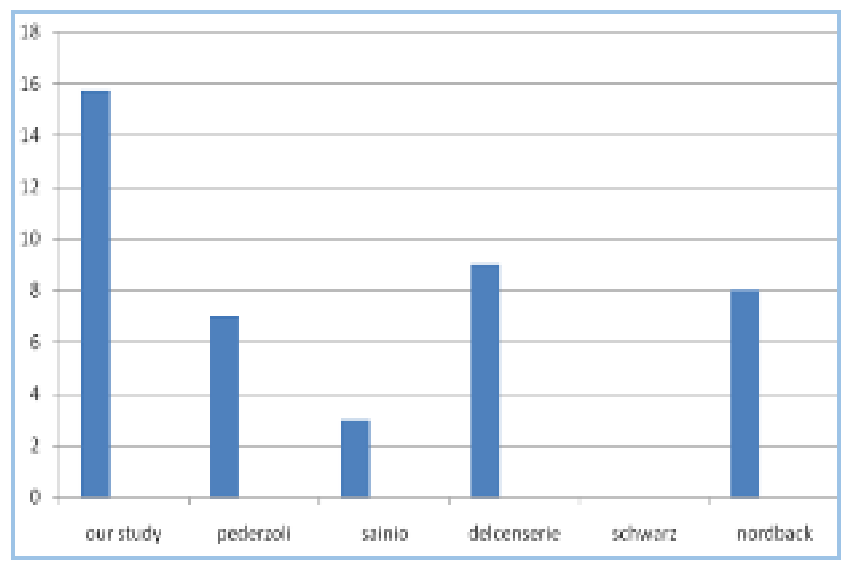

Fig. 17: Prophylactic Antibiotics and Mortality in ANP

\section{\% DEATH RATE}

Balthazar's Score and Mortality

Patients with score 7-10 had more mortality compared to standard may be due to study in referral from other hospital.

\begin{tabular}{|c|c|c|c|c|}
\hline Score & Frequency & $\begin{array}{c}\text { Death } \\
\text { Rate }\end{array}$ & $\begin{array}{c}\text { \% } \\
\text { Death } \\
\text { Rate } \\
\text { (Our } \\
\text { Study) }\end{array}$ & $\begin{array}{c}\text { \% Death } \\
\text { Rate } \\
\text { (Balthazar's } \\
\text { Study) }\end{array}$ \\
\hline $0-3$ & 49 & 0 & $0 \%$ & $3 \%$ \\
\hline $4-6$ & 25 & 1 & $3.33 \%$ & $6 \%$ \\
\hline $7-10$ & 31 & 7 & $22.5 \%$ & $17 \%$ \\
\hline \multicolumn{4}{|c|}{ Table 18: Balthazar's Score and Mortality } \\
\hline
\end{tabular}

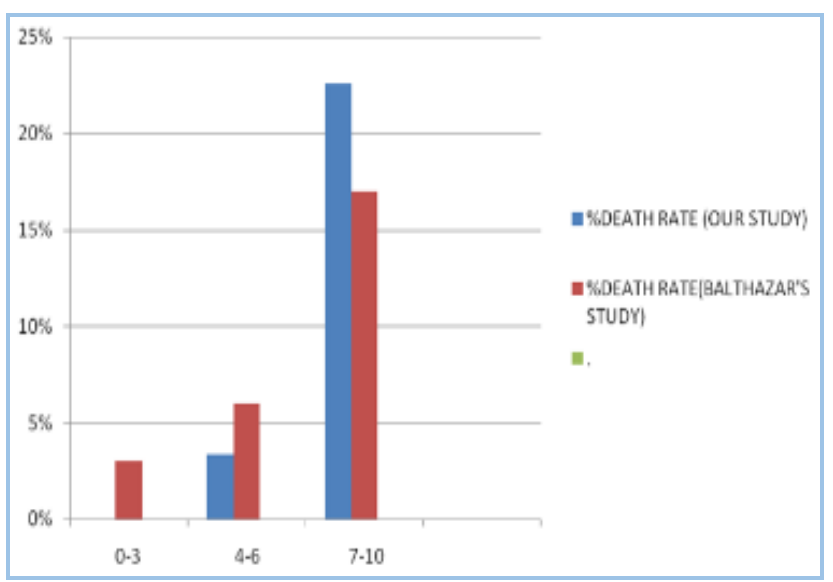

Fig. 18: Balthazar's Score and Mortality

Mortality

\begin{tabular}{|c|c|c|c|c|}
\hline Reference & Country & $\begin{array}{c}\text { All } \\
\text { Cases }\end{array}$ & $\begin{array}{c}\text { Death } \\
\text { Rate }\end{array}$ & $\begin{array}{c}\text { \% Death } \\
\text { Rate }\end{array}$ \\
\hline Mann et al. & UK & 631 & 57 & $9.0 \%$ \\
\hline $\begin{array}{c}\text { Talamini } \\
\text { et al. }\end{array}$ & Italy & 192 & 17 & $8.9 \%$ \\
\hline $\begin{array}{c}\text { Mutinga } \\
\text { et al. }\end{array}$ & USA & 805 & 17 & $2.1 \%$ \\
\hline Blum et al. & Germany & 351 & 17 & $4.8 \%$ \\
\hline Floyd et al. & Denmark & 480 & 44 & $9.2 \%$ \\
\hline $\begin{array}{c}\text { National } \\
\text { Survey }\end{array}$ & Japan & 1240 & 92 & $7.4 \%$ \\
\hline Our Study & & 105 & 8 & $7.61 \%$ \\
\hline \multicolumn{5}{|c|}{ Table 19: Comparison of Mortality from Acute } \\
Pancreatitis-Our's Vs. Other Studies \\
\hline
\end{tabular}

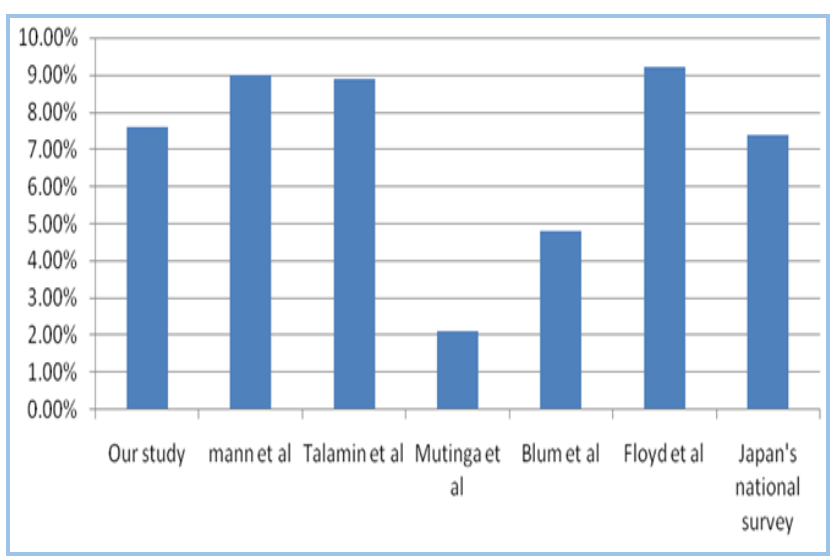

Fig. 19: Comparison of Mortality from Acute Pancreatitis-Our's Vs. Other Studies 


\begin{tabular}{|c|c|c|c|}
\hline References & Year & $\begin{array}{c}\text { Death } \\
\text { Rate }\end{array}$ & \% Death Rate \\
\hline Bradley and Allen & 1991 & 38 & $15.0 \%$ \\
\hline Allardyce & 1987 & 17 & $80.0 \%$ \\
\hline Perez et al. & 2002 & 99 & $14.0 \%$ \\
\hline Gull et al. & 2002 & 479 & $16.0 \%$ \\
\hline $\begin{array}{c}\text { Japan's National } \\
\text { Survey }\end{array}$ & 2000 & 117 & $23.0 \%$ \\
\hline Our Study & $\mathbf{3}$ & $\mathbf{1 5 . 7 \%}$ \\
\hline $\begin{array}{c}\text { Table 20: Comparison of Mortality from Acute } \\
\text { Necrotizing Pancreatitis-Our's Vs. Other Studies }\end{array}$ \\
\hline
\end{tabular}

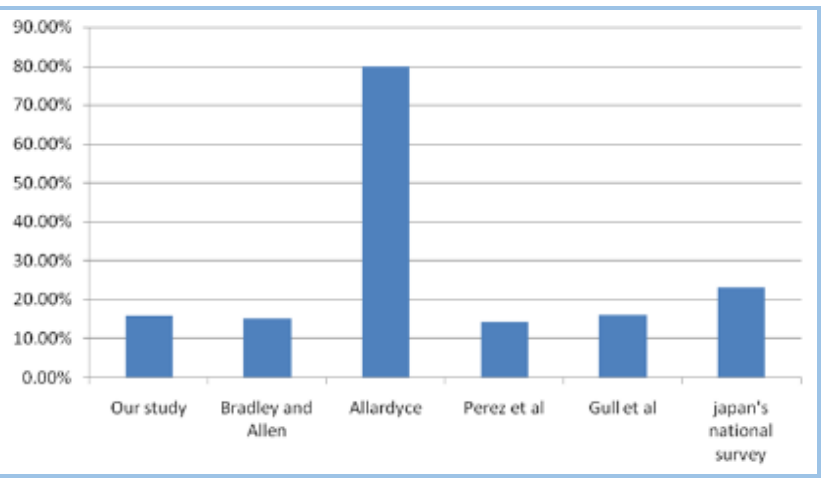

Fig. 20: Comparison of Mortality from Acute Necrotizing Pancreatitis-Our's Vs. Other Studies

\section{SUMMARY}

This prospective study conducted at Mahatma Gandhi Memorial Hospital, Warangal, included 105 patients with acute pancreatitis, 75 males and 30 females. The peak incidence was in the fourth decade with the median age of 32 years. Alcohol is the most common aetiology followed by gall stones.

Serum Amylase and Serum Lipase together gave high sensitivity $(80 \%)$ for diagnosis. Computed tomography was very sensitive than ultrasonography, non-invasive tool for diagnosis and imaging of complications. Early enteral nutrition was started in all patients of pancreatitis within 48 hours. Most common local complication was acute fluid collection. The median hospital stay was 12 days (Range- 3 to 85 days) and 23 patients required ICU care ( 2 to 56 days).

Out of 105 patients, $18.09 \%$ had acute necrotizing pancreatitis. The overall mortality rate was $7.61 \%$ and mortality rate among acute necrotizing pancreatitis cases was $15.7 \%$.

\section{CONCLUSION}

The incidence of acute pancreatitis was found to be in a younger age group in our study. Serum Amylase and Lipase both (80\% sensitivity) should be used for diagnosis wherever possible. Ideally, all cases should be stratified during the first 48 hours according to one of the scoring systems (Balthazar's). Scoring systems help to identify patients who are more likely to have a severe attack and they should be referred to higher centres if adequate facilities are not available.

Enteral nutrition should be started in all patients of acute pancreatitis within 48 hours. Prophylactic antibiotics decrease septic complications in severe acute pancreatitis.
Severe cases should be managed in well-equipped ICU, since they may require massive fluid resuscitation, mechanical ventilation and haemodialysis. Support of specialist in Radiology, Endoscopy and Surgery is essential.

Timely intervention by endoscopist and surgeons are crucial to reduce morbidity and mortality. Further attacks should be prevented by early cholecystectomy and avoiding alcohol.

\section{REFERENCES}

1. Opie EL. The etiology of acute haemorrhagic pancreatitis. Bull Johns Hopkins Hosp 1901;12:182-188.

2. Baron TH, Morgan DE. Current concepts: acute necrotizing pancreatitis. N Engl J Med 1999;340(18):1412-1417.

3. Steinberg W, Tenner S. Acute pancreatitis. N Engl J Med 1994;330(17):1198-1210.

4. Dulce M Cruz-Santamaria. Update pathogenesis and clinical management of acute pancreatitis. World J Gastrointest Pathophysiol 2012;3(3):60-70.

5. Norman S Williams, Christopher JK Bulstrode, Ronan O'Connell. Bailey and Love short textbook of surgery. London: Hodder Arnold, 2009;25 th Ed:1130-54.

6. Yeo. Shackelford's surgery of alimentary tract. Saunders, 2007; $6^{\text {th }}$ ed.

7. Toh SK, Phillip S, Johnson CD. A prospective audit against national standards of the presentation and management of acute pancreatitis in the south of England. Gut 2000;46(2):239-243.

8. Miho Sekimoto, Tadahiro Takada, Yoshifumi Kawarada, et al. JPN guidelines for the management of acute pancreatitis: epidemiology, etiology, natural history and outcome predictors in acute pancreatitis. J Hepatobiliary Pancreat surg 2006;13:10-24.

9. Rudra Prasad Doley, Thakur Deen Yadav, Jai Dev Wig, et al. Enteral nutrition in severe acute pancreatitis. JOP J pancreas (Online) 2009;10(2):157-162.

10. Pederzoli P, Bassi C, Vesentini S, et al. A randomized multicenter clinical trial of antibiotic prophylaxis of septic complications in acute necrotizing pancreatitis with imipenem. Surg Gynecol Obstet 1993;176:480-3.

11. Sainio V, Kemppainen E, Puolaikken P, et al. Early antibiotic treatment in acute necrotizing pancreatitis. Lancet 1995;346:663-7.

12. Delcenserie R, Yzet T, Ducroix JP. Prophylactic antibiotics in treatment of severe acute alcoholic pancreatitis. pancreas 1996;13:198-201.

13. Schwartz M, Isenmann $\mathrm{R}$, Meyer $\mathrm{H}$, et al. Antibiotic use in necrotizing pancreatitis. Results of a controlled study. Dtsch Med Wochenschr 1997;122:356-61.

14. Nordback I, Sand J, Saaristo R, et al. Early treatment with antibiotics reduces the need for surgery in acute necrotizing pancreatitis-a single-center randomized study. J Gastrointest surg 2001;5:113-118.

15. Isenmann R, Runzi M, Kron $M$, et al. Prophylactic antibioyic treatment in patients with predicted severe acute pancreatitis: a placebo-controlled, double-blind trial. Gastroenterology 2004;126:1997-1004. 